\title{
Les Intoxications Volontaires Par Les Pesticides Dans La Région De Rabat-Salé -Zemmour- Zaer Entre 2008 Et 2014
}

\author{
Detsouli Aicha \\ Institut agronomique et vétérinaire Hassan II, Rabat, Maroc \\ Abidli Zakaria \\ Kharbouch Donia \\ Hmimou Soumaia \\ PPR-B-Mokhtari-FS-UIT-Kenitra, Laboratoire de Génétique et Biométrie, \\ Faculté des Sciences, Université Ibn Tofail, Kénitra, Maroc
}

\section{Amiar Latifa}

Faculté des Sciences et Techniques de Tanger

Soulaymani Abdelmajid

\section{Mokhtari Abdelrhani}

PPR-B-Mokhtari-FS-UIT-Kenitra, Laboratoire de Génétique et Biométrie,

Faculté des Sciences, Université Ibn Tofail, Kénitra, Maroc

\section{Soulaymani- Bencheikh Rachida}

Centre Anti Poison et de Pharmacovigilance du Maroc

Université Mohammed V, Rabat, Maroc

Doi: 10.19044/esj.2018.v14n15p317 URL:http://dx.doi.org/10.19044/esj.2018.v14n15p317

\begin{abstract}
The objective of this study is to describe the epidemiological characteristics and the risk factors for voluntary poisoning by pesticides in the Rabat-Salé-Zemmour-Zaer region between 2008 and 2014. This is a retrospective study of cases of voluntary poisoning by pesticides collected between 2008 and 2014 by the Anti-poisoning and Pharmaco-vigilance Center of Morocco. During the study period, 598 cases of voluntary pesticide intoxication were collected. These voluntary intoxications mainly concerned adults whose age is between 20 and 74 years. Female addicts dominated the scene with $61.87 \%$ and a sex ratio $(\mathrm{F} / \mathrm{H})$ of 2.02 in favor of female sex, with urban predominance. The risk was related to the use of raticides (195 cases). The evolution was favorable in $94 \%$ of cases, with 27 people dying during this period.
\end{abstract}


Keywords: Voluntary poisoning, pesticides, Anti-poisoning and Pharmacovigilance Center of Morocco, Rabat-Salé-Zemmour-Zaer region

\section{Résumé}

L'objectif de cette étude est de décrire les caractéristiques épidémiologiques et de déterminer les facteurs de risque des intoxications volontaires par les pesticides dans la région de Rabat-Salé -Zemmour- Zaer entre 2008 et 2014. Il s'agit d'une étude rétrospective descriptive des cas d'intoxication volontaire par les pesticides colligé entre 2008 et 2014 par le Centre Anti Poison et de Pharmacovigilance du Maroc. Au cours de la période d'étude, 598 cas d'intoxication volontaire par les pesticides ont été collectés. Ces intoxications volontaires ont concerné principalement les adultes dont l'âge est compris entre 20 ans et 74 ans. Les intoxiqués du sexe féminin ont prédominé la scène avec $61.87 \%$ et un sexe ratio $(\mathrm{F} / \mathrm{H})$ de 2.02 en faveur du sexe féminin, avec une prédominance urbaine .Le risque était lié à l'utilisation des raticides (195 cas). L'évolution a été favorable dans 94\% des cas, avec 27 personnes ont décédé durant cette période.

Mots Clés : Intoxications volontaires, pesticides, Centre Anti Poison et de Pharmacovigilance du Maroc, région de Rabat-Salé -Zemmour- Zaer

\section{Introduction}

Les intoxications par les pesticides de toutes sortes soient accidentels ou volontaires représentent un problème majeur de santé publique. Selon le rapport de l'Organisation Mondiale de la Santé (OMS), le nombre annuel d'intoxications par les pesticides est estimé entre 1 et 5 millions, dont plusieurs milliers de cas sont mortels (OMS 2004). En effet, Au niveau national, le Centre Anti Poison et de Pharmacovigilance du Maroc (CAPM), déclare que les intoxications aigues aux pesticides (IAP) occupent la $4^{\text {éme }}$ position après les médicaments, les produits industriels et les aliments avec un taux brut d'incidence de 2,56 pour 100000 habitants, dont 23,1\% d'intoxications aux pesticides sont volontaires (Ait El Cadi 2009). Au niveau régional, une étude rétrospective réalisée sur une série de 500 malades admis à l'hôpital d'enfants de Rabat, les pesticides étaient responsables de $38 \%$ des cas d'intoxication aiguës (Madani $\mathrm{N}$ et coll). Au niveau volontaire une étude a démontré que le taux le plus élevé d'intoxication volontaire par les pesticides a été enregistré dans la région de Rabat-SaleZemmour-Zaer 598 cas, suivie de la région de Meknès-Tafilalt avec 332 cas (Zineb Nabih et coll).

En ce sens, Les objectifs de ce travail consistent donc à présenter les aspects épidémiologiques, cliniques et évolutifs des intoxications volontaires par les pesticides dans la région de Rabat-Salé-Zemmour-Zaer entre 2008 et 
2014 en vue de promouvoir des actions pour leur prise en charge médicale et leur prévention.

\section{Données et Méthodes}

Il s'agit d'une étude épidémiologique rétrospective, portant sur tous les cas d'intoxications volontaires par les pesticides survenus dans la région du Rabat-Salé-Zemmour-Zaër, déclarés à l'unité d'information toxicologique du CAPM sur une durée de Sept ans, allant de janvier 2008 à décembre 2014.

La région de Rabat-Salé-Zemmour-Zaër se trouvait géographiquement au nord-ouest du pays. Sa superficie était de $9580 \mathrm{~km} 2$ avec une population de 2366494 habitants (recensement officiel de l'année 2004) représentant $7,9 \%$ de la population totale du royaume, avec un taux d'accroissement annuel moyen de $1,8 \%$ contre $1,4 \%$ au niveau national (Haut Commissariat au plan (Maroc),2011). Ils représentent l'une des seize régions que le Maroc comportait avant l'ancien découpage territorial de 2015.Sur le plan administratif, elle est découpée en quatre provinces : Rabat,Sale,SkhiratTemara et Khemisset.(carte Sig).

Les variables étudiées concernent les caractéristiques de la population intoxiquée (année, sexe, âge, origine, type d'intoxication, circonstance, symptomatologie, gradation et évolution), les caractéristiques du toxique (type de pesticide en cause). L'âge a été analysé selon classification INTOX. L'évaluation de la gravité s'est faite par le poisoning score severity (PSS) (Person 1998) (tableau 1)

Tableau 1 : L'évaluation de la gravité s'est faite par le poisoning score severity (PSS)

(Person 1998)

\begin{tabular}{cc}
\hline Grade & Les signes \\
\hline 0 & Absence de signe fonctionnel ou physique \\
1 & Symptômes mineurs, transitoires et régressant spontanément \\
2 & Symptômes marqués ou persistants \\
3 & Symptômes sévères ou engageant le pronostic vital \\
4 & Intoxication mortelle \\
\hline
\end{tabular}

En effet, La méthodologie statistique s'est basée sur deux axes : la statistique descriptive et la statistique analytique. Dans un premier volet, on à dégager les fréquences et les caractéristiques de chaque variable étudiée ce qui nous a permis de dresser un profil épidémiologique de tous les cas d'intoxication. Les résultats ont été exprimés en pourcentage pour les variables qualitatives et en moyenne \pm écart-type pour les variables quantitatives. Dans un deuxième temps, on a utilisé le test $\chi^{2}$ qui permettre de savoir si une différence significative existe entre certaines variables et le calcul $\mathrm{du}$ risque relatif nous ont permis d'étudier l'association entre les variables étudiées et l'évolution. La létalité a été également calculée afin de connaitre avec précision les gravités de la problématique au niveau de cette région. 


\section{Résultats}

Il s'agit d'une étude de 598 cas d'intoxication volontaires par les pesticides signalés par le CAPM durant la période d'étude, dans la région Rabat-Salé-Zemmour- Zaer. La figure ci-dessous montre la distribution des cas d'intoxications et de la létalité spécifique selon les années.

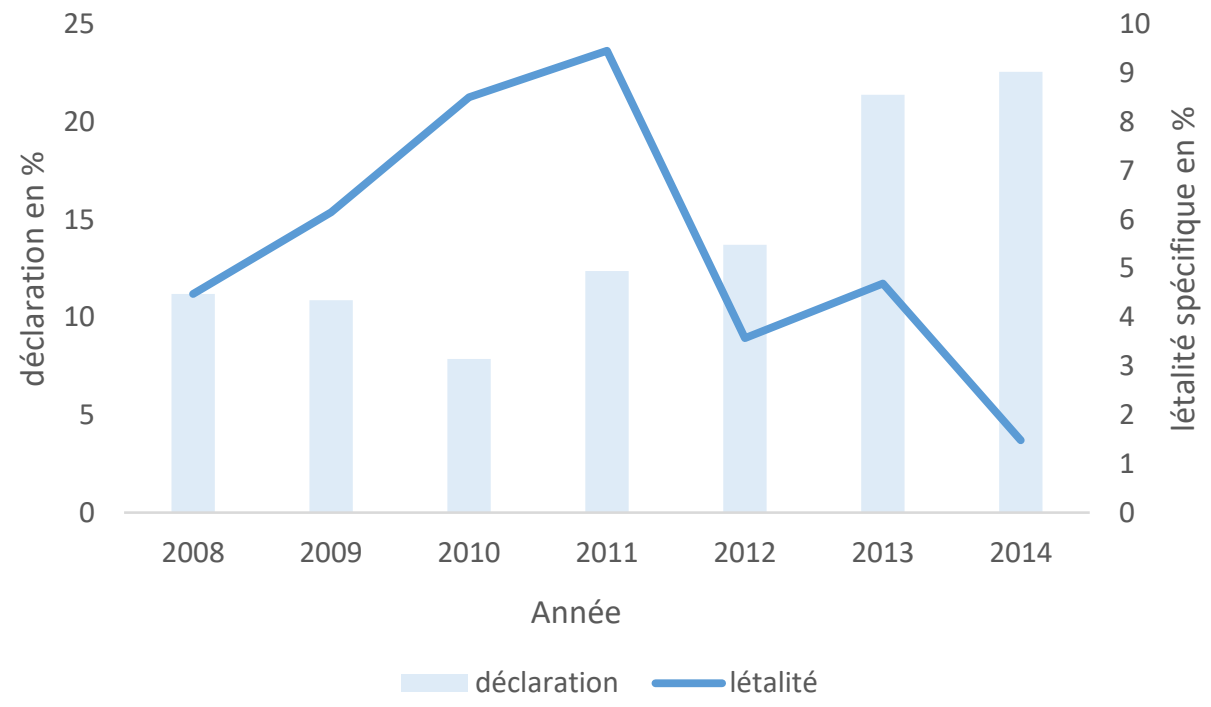

Figure1 : Distribution des cas d'intoxication et de la létalité spécifique selon les années

Les déclarations des cas étaient généralement progressives et évolutives, depuis l'année 2008 avec un pic en 2014 (21.40\%), tandis que la létalité spécifique à était signalé en 2011 avec un pourcentage de $9.45 \%$.

Tableau 2: Répartition des intoxications en fonction des caractéristiques du patient et de l'intoxication

\begin{tabular}{|c|c|c|c|c|c|}
\hline \multirow[t]{2}{*}{ Variables } & \multirow[t]{2}{*}{$\mathrm{N}$ cas } & \multicolumn{3}{|c|}{ Évolution } & \multirow[t]{2}{*}{ LS \% } \\
\hline & & Guérison & Décès & Inconnu & \\
\hline \multicolumn{6}{|c|}{ Sexe } \\
\hline Féminin & 370 & 256 & 17 & 97 & 4.59 \\
\hline Masculin & 183 & 116 & 8 & 59 & 4.37 \\
\hline Inconnu & 45 & 22 & 2 & 21 & 4.44 \\
\hline \multicolumn{6}{|c|}{ Groupes d'âge } \\
\hline Nouveau-né & 0 & 0 & 0 & 1 & \\
\hline Bébé marcheur & 3 & 2 & 0 & 1 & 0 \\
\hline Enfants & 40 & 32 & 2 & 6 & $\begin{array}{l}0 \\
5\end{array}$ \\
\hline Adolescents & 139 & 96 & 4 & 39 & 1.43 \\
\hline Adultes & 410 & 262 & 20 & 128 & 4.80 \\
\hline Personnes âgées & 1 & 0 & 0 & 1 & 0 \\
\hline Inconnu & 5 & 2 & 0 & 3 & 0 \\
\hline \multicolumn{6}{|c|}{ Gradation (PSS) } \\
\hline Grade 0 (Néant) & 140 & 131 & 0 & 9 & - \\
\hline
\end{tabular}




\begin{tabular}{|c|c|c|c|c|c|}
\hline & & & & & \\
\hline Grade 1 (Mineur) & 78 & 71 & 0 & 7 & - \\
\hline Grade 2 & 192 & 155 & 0 & & - \\
\hline (Modérés) & & & & 37 & \\
\hline $\begin{array}{l}\text { Grade } 3 \\
\text { (Sévères) }\end{array}$ & 30 & 24 & 0 & 6 & - \\
\hline Grade 4 (Fatal) & 27 & 0 & 27 & 0 & 100 \\
\hline inconnu & 131 & 14 & 0 & 117 & - \\
\hline & & & & & \\
\hline Urbain & & 336 & 21 & 112 & 4.89 \\
\hline & 429 & & & & \\
\hline Rural & & 71 & 2 & & 1.81 \\
\hline & 110 & & & 37 & \\
\hline Inconnu & & 27 & 4 & & 6.77 \\
\hline & 59 & & & & \\
\hline & & & & 28 & \\
\hline Lieu & & & & & \\
\hline Domicile & 504 & 336 & 18 & 150 & 3.5 \\
\hline Public & 7 & 5 & 0 & 2 & 0 \\
\hline Travail & 1 & 3 & 0 & 2 & 0 \\
\hline Prison & 1 & 1 & 0 & 1 & 0 \\
\hline Inconnu & 85 & 51 & 9 & 25 & 10.58 \\
\hline & & & & & \\
\hline Asymptomatique & & 154 & 6 & 70 & 2.60 \\
\hline & 230 & & & & \\
\hline Symptomatique & 368 & 240 & 21 & 107 & 5.70 \\
\hline & & Type de & estici & & \\
\hline Herbicides & 5 & 4 & 0 & 1 & 0 \\
\hline Insecticides & 132 & 90 & 3 & 39 & 2.27 \\
\hline Raticides & 195 & 139 & 6 & 50 & 3.07 \\
\hline Inconnu & 266 & 162 & 18 & 86 & 6.76 \\
\hline
\end{tabular}

L.S : létalité spécifique, PSS : Poisoning Score Severity,

L'âge moyen de la population intoxiquée était de 24.01士9.45ans. Les intoxications volontaires ont concerné principalement les adultes dont l'âge est compris entre 20 ans et 74 ans (environ 68.56\%). Les intoxiqués du sexe féminin ont prédominé la scène avec $61.87 \%$ et un sexe ratio $(\mathrm{F} / \mathrm{H})$ de 2.02 en faveur du sexe féminin La plupart des intoxiqués qui ont déclaré leurs origines, étaient urbain (80\% des cas). La différence entre les deux origines (urbain, rural) a été hautement significative $(\mathrm{p}=0.001)$. La répartition géographique montre que les quatre provinces ont été touchées par ce fléau, en outre, le maximum de déclarations a été enregistré dans la province de Rabat $(62.5 \%$ des cas) avec une létalité plus élevée de 5.3\%. Trios familles des pesticides étaient identifiéses dans notre série de données. les intoxications par les raticides étianent les plus importantes (32. 6\%) par rapport autres types du pesticide. Pour ce qui concerne la circonstance, L'acte suicidaire est à l'origine 
de 98,49\% des intoxications volontaires par les pesticides. Cependant, les intoxications dont l'objectif criminel, avortement et toxicomanie représentent des faibles pourcentages, mais la létalité la plus importante a été marquée dans le cadre criminel (25\%). L'état clinique des intoxiqués était en majorité symptomatique avec un pourcentage de $62 \%$ des cas. La plupart des intoxiqués ont présenté un état modéré (Garde 2) avec 32.10\%, suivi par l'absence de signe fonctionnel ou physique (Garde 0) avec 23.1\%, l'état mineur (Garde 1) représente $13.04 \%$ des cas, et finalement on trouve l'état fatale, on parle donc de 27 cas de décès $(4.51 \%)$. Le système nerveux central et périphérique est le chef du fil des systèmes affectés avec $52.66 \%$ des cas, avec une létalité spécifique de 7,5\% (Le nombre de décès étant de 6 sujets). Les patients ont présenté plusieurs signes neurologiques dont des Convulsions et crises cloniques, des Obnubilations, Agitation, Céphalée, et Coma respectivement avec $17,61 \% ; 10,56 \% ; 7,75 \% ; 7,04 \% ; 6,34 \%$ des cas. Pour l'évolution a été favorable dans 94\% des cas. Malheureusement 27 personnes ont décédé durant cette période.

Tableau 4. Effet des caractéristiques étudiées sur l'évolution de l'état de santé des patients

\begin{tabular}{ccccccc}
\hline Variables & Modalité & Guérison & Décès & $\mathrm{X}^{2}$ & $\mathrm{RR}$ & $\mathrm{IC95 \%}$ \\
\hline \multirow{2}{*}{ Sexe } & Masculin & 116 & 8 & \multirow{2}{*}{0.54} & \multirow{2}{*}{0.93} & $0.44-2.29$ \\
& Féminin & 256 & 17 & & & \\
Age & $>15$ & 358 & 24 & 0.72 & 0.71 & $0.21-2.56$ \\
& $<=15$ & 34 & 2 & & & \\
Milieu & Urbain & 336 & 21 & 0.27 & 2.51 & $0.75-10.91$ \\
& Rural & 71 & 2 & & & \\
Clinique & Symptomatique & 240 & 21 & 2.100 & 0.101 & $0.886-1.59$ \\
& Asymptomatique & 154 & 6 & & & \\
\hline
\end{tabular}

Selon les résultats du tableau, on note que l'âge de l'intoxiqué, les circonstances de l'intoxication et le produit toxique en cause sont des facteurs de risque qui avaient influencés significativement sur l'évolution de l'état de santé des patients intoxiqués volontairement par les pesticides. Les intoxiqués appartenant au milieu urbain ont 2.5 de risque de décès que ceux appartenant au milieu rural $(\mathrm{RR}=2.5$; IC $95 \%: 0.75-10.91)$

\section{Discussion}

Au Maroc, comme dans beaucoup de pays du monde, les intoxications volontaires par les pesticides sont responsables chaque année d'un grand nombre de décès et les placent parmi les principaux enjeux de santé publique marocaine. Selon les résultats déclarés au CAPM, la région de Rabat-SaléZemmour-Zaë a enregistré le taux le plus élevé des intoxications volontaires par les pesticides avec une incidence de 3.14 pour 100000 habitants (Zineb Nabih et coll ,2017). Ces résultats confirment que les intoxications volontaires aux pesticides dans cette région sont loin d'être négligeables. 
En effet, dans notre étude, nous avons recensé 598 cas d'intoxication volontaire par les pesticides dans la région du Rabat-Salé-Zemmour-Zaër entre 2008 et 2014. L'acte suicidaire est à l'origine de 98,49 \% des intoxications volontaires par les pesticides. Cependant, les intoxications dont l'objectif criminel, avortement et toxicomanie représentent des faibles pourcentages. Une étude effectuée dans la région de Tanger-Tétouan a démontré que la circonstance accidentelle dominait avec $81 \%$ des cas, les cas volontaires sont plus au moins significatifs (19\% des cas) dont les cas de tentatives de suicide représentent 17\% (Toilabiya et al, 2012). Une étude faite à Bamako par Traoré (2006) qui a trouvé que 2 sur 3 des cas d'intoxications sont volontaires (Traoré, 2006). Par malheur, l'acte suicidaire reste un sujet tabou et très mal juger à cause de plusieurs facteurs dans la société africaine.

En outre, d'après la revue de la littérature, nous constatons que les pesticides sont l'un des outils les plus utilisés dans les tentatives suicidaires. Une étude effectuée au Maroc par Amiar en 2016 qui a trouvé que les pesticides occupe la deuxième position dans la famille du toxique la plus utilisée comme produit suicidaire avec un pourcentage de 30,5\% (Amiar Latifa, 2016). Dans notre série de cas, les pesticides étaient impliqués dans 587 cas d'intoxication dont l'objectif suicidaire (engendrant 26 décès).Cette résultat concordant avec la littérature (OMS, 2002).

Dans notre étude, les intoxications suicidaires ont touché les deux sexes avec une prédominance féminine. Nos résultats concordent avec ceux de nombreuses études, au niveau national et international (Ouammi et al. 2009 ; Loutfiya et al, 2012 ; Mahir et al,2013 ;Diallo T,2013 Amiar Latifa,2016 et Nabih,2017). Il est important de noter que les filles auraient plus souvent des idéations suicidaires que les garçons (Crosby et al. 1994). Des études épidémiologiques sur le suicide ont mis en évidence des différences significatives en termes de morbidité et de mortalité du geste suicidaire en fonction du sexe. Le risque de mortalité pour les garçons est plus important que pour les filles. Cette différence peut être expliquée par le choix du produit toxique et aussi par la dose ingérée qui varie selon les deux sexes (Staikowsky et al, 1995).

L’âge moyen de la population intoxiquée était de $24.01 \pm 9.45$ ans. Ce sont les adultes et les adolescents qui ont été les plus impliqués dans les intoxications volontaires surtout dans le cadre suicidaire. D'après N. Rhalem, 2009 et Safae El khaddam, 2012, ce résultat pourrait être expliqué par le jeune âge de la population marocain et la confrontation des jeunes aux difficultés de la vie et aux problèmes liés aux conflits familiaux, conjugaux, sentimentaux, échecs scolaires mais surtout le chômage. Les tentatives de suicide impliquant le plus souvent des adolescents étaient signalées par (Jeffery et al. en 2006, Amiar Latifa, 2016) 
Dans cette étude, les raticides constituent le chef de file des produits en cause dans les intoxications volontaires par pesticides 195 cas, suivis des insecticides avec 132 cas. Ces résultats sont comparables à ceux de la plupart des études publiées (Benjabblah 1994, Jouglard 1992), selon la littérature, les intoxications par les insecticides organophosphorés sont mortelles dans 4 à 30 $\%$ des cas (Achour, 2011).

Sur le plan clinique, Le système nerveux central et périphérique est le chef du fil des systèmes affectés avec $52.66 \%$ des cas, avec une létalité spécifique de 7,5\% avec 6 sujets décès. Cependant, de nombreux travaux ont montré que plusieurs types des pesticides exercent une action neurotoxique chez l'individu et qu'un lien potentiel entre l'exposition à certains pesticides et la maladie de Parkinson pourra exister, suite à l'augmentation de marqueurs du stress oxydant, en plus à autres dégénérescences neuronales et anomalies du développement (Mccormack 2002).

L'évolution clinique des intoxiqués volontairement par les pesticides a été généralement favorable, et le décès est survenue dans 27 cas. D'après la revue de la littérature la fréquence varie de 4 à 25\% (S. B. Agarwal, 1993).

\section{Conclusion}

D'après nos résultats, les intoxications par les pesticides sont responsables d'effets néfastes sur la santé. En outre, les autorités doivent réglementer l'achet des pesticides pour diminuer le taux d'intoxications volontaire au Maroc.

\section{References:}

1. Achour S, Rhalem N., Khattab A. (2011). Les caractéristiques cliniques et thérapeutiques des intoxications par les organophosphorés : données du Centre Antipoison et de pharmacovigilance du Maroc. Santé publique.p-5.6.

2. Agarwal S. B, "A clinical, biochemical, neurobehavioral, and sociopsychological study of 190 patients admitted to hospital as a result of acute organophosphorus poisoning," Enviromental Research, vol. 62, n 1, pp. 63-70, 1993.

3. Ait El cadi M.,Mezzane A.,Meddah B., Khabbal Y., khabbal.,Idrissi M.(2009). Intoxications mortelles aux pesticides au Maroc (20002005), Epidémiologie et santé publique, (vol 57), pp. 6.

4. AMIAR Latifa, Abdelmajid SOULAYMANI, Ahmed AARAB, Abdelrhani MOKHTARI, Rachida SOULAYMANI (2016). Aspects Épidémiologiques des Suicides et des Tentatives de Suicide par Intoxication au Maroc. European Journal of Scientific Research Vol. 140 No 3 July, 2016, pp.282-293

5. Benjabblah N., Trabelsi M., Khaldi F., Ouerghi M., Bennaceur 
B.(1994). Les intoxications aiguës accidentelles chez l'enfant (à propos de 281 cas). Maghr pédiatrie. (Vol 4).p87-91.

6. Crosby, FJ (1994). Comprendre l'action positive. Basique et appliqué Psychologie sociale, 15, 13-41

7. Diallo T., Hami H., Maiga A., CoulibalyB., Maiga D., Mokhtari A., Soulaymani R. et Soulaymani A. Introduction Épidémiologie et facteurs de risque des intoxications volontaires au Mali. Santé Publique. Volume 25. N³. mai-juin 2013359 - 366.

8. Haut-Commissariat au plan (Maroc), Recensement général de la population et de 1'habitat de 2004, Caractéristiques démographiques et socio-économiques : Rabat-Salé-Zemmour-Zaër, 11, 2011.

9. Jeffrey A. Bridge, Tina R. Goldstein and David A. Brent. Adolescent suicid and suicidal behavior Western Psychiatric Institute and Clinic, University of Pittsburgh Medical Center, Pittsburgh, PA, USA. Journal of Child Psychology and Psychiatry. 2006; Vol.47 : N 3 -4, 372-394

10. Joint note for the media WHO/FAO/UNEP, Children are fac-ing high risks from pesticide poisoning, 24 September 2004,Geneva.http://www.who.int.

11. Jouglard J. (1992). Epidémiologie des intoxications avec étude des principaux produits ingérés. Communication des experts ,Conférence de Consensus en réanimation.

12. Madani $\mathrm{N}$ et coll. Les intoxications aigues admises en réanimation (à propos de 500 cas). Maghreb Médical 2000; 20(349): 264-268.

13. Mahir S., Soulaymani A., Hami H., Mokhtari A., Benali D., Ouammi L., Windy M. et Soulaymani R. Suicides par intoxication dans la région de Souss-Massa-Drâa au Maroc. Santé publique volume 25. N³ - mai-juin 2013. 343-350.

14. Mccormack AL., Thiruchelvam M., Manning-Bog A., Thiffault C, Langston JW., Cory-Slechta DA., Di Monte DA. (2000). Environmental risk factors and Parkinson's disease: selective degeneration of nigral dopaminergic neurons caused by the herbicide paraquat. Neurobiol. (vol10).p-27.

15. Nabih Zineb, Latifa Amiar, Zakaria Abidli, Maria Windy, Abdelmajid Soulaymani, Abdelrhani Mokhtari, Rachida Soulaymani-Bencheikh Epidemiology and risk factors of voluntary pesticide poisoning in Morocco (2008-2014).Epidemiol Health. 2017 Sep 1;39

16. Ouammi L., N. Rhalem, R. Aghandous, I. Semllali, M. Badri, G. Jalal, S. Benlarabi, A. Mokhtari, A. Soulaymani, and R. SoulaymaniBenchikh, "Profil épidémiologique des intoxications au Maroc de 1980 à 2007', Toxicologie Maroc, vol. 1, pp. 8-13, 2009

17. Rhalem N., A. Khattabi, S. Achour, A. Soulaymani, and R. Soulaymani Bencheikh, "Facteurs prédictifs de gravité de 
l'intoxication aux pesticides - Expérience du Centre Antipoison du Maroc," Annales de Toxicologie Analytique, vol. 21, $\mathrm{n}^{\circ}$ 2, pp. 79-84, 2009

18. Safae El khaddam, Moncef Idrissi, Sanae Achour, Abd Errazak Khadmaoui, Fatine Hadrya, Abdelmajid Soulaymani, Rachida Soulaymani-Bencheikh (2013). Les intoxications aiguës aux pesticides dans la région de Tadla-Azilal (Maroc): Évolution et facteurs de risque. International Journal of Innovation and Applied Studies ISSN Vol. 3 No. 2 June 2013, pp. 552-559

19. Staikowsky, F., Uzan, D., Grillon, N., Pevirieri, F., Hafi, A., Michard, F. Intoxications medicamenteuses volontaires reçues dans un service d'accueil des urgences. Presse Médicale, pp 1296-1300, 1995 